\title{
Effect of CNT Length and Structural Density on Viscoelasticity of Buckypaper: A Coarse-Grained Molecular Dynamics Study
}

\author{
Heng Chen ${ }^{a b}$, Liuyang Zhang ${ }^{b}$, Jinbao Chen ${ }^{a}$, Matthew Becton ${ }^{b}$, \\ Xianqiao Wang ${ }^{b}$, and Hong $\mathrm{Nie}^{a}$ \\ ${ }^{a}$ College of Aerospace Engineering, Nanjing University of Aeronautics and Astronautics, \\ Nanjing, Jiangsu 210016 China \\ ${ }^{b}$ College of Engineering, University of Georgia, Athens, GA 30602 USA
}

\begin{abstract}
Carbon nanotube (CNT) buckypapers, having exceptional mechanical and electrical properties, have been reported to demonstrate frequency-invariant and temperature-invariant viscoelastic properties. In an attempt to provide an in-depth insight into the viscoelasticity of buckypapers with $(5,5)$ single-walled CNTs (SWCNTs), we perform coarse-grained non-equilibrium molecular dynamics simulations to investigate the effects of oscillatory shear strain amplitude, buckypaper's density, and length of individual SWCNTs on the viscoelastic properties. SWCNT buckypapers exhibit linear viscoelasticity over shear strain amplitude from 0.03 to 0.05 . Higher density SWCNT buckypapers can result in larger dynamic stiffness and a higher loss factor of up to $\sim 0.29$. In the frequency-independent regime ( $\leq 1 \mathrm{GHz}$ ), increasing the length of individual SWCNTs causes a very slight decrease of elastic properties and has minor influence on the viscous mode. Thus, this study provides deep insight into the viscoelasticity of $(5,5)$ SWCNT buckypapers and demonstrates controllability of the excellent energy dissipation potential of buckypapers, and can thus help us design new energy dissipation devices from carbon nanomaterials.
\end{abstract}




\section{Introduction}

Carbon nanotubes (CNTs) have shown unprecedented thermal, electrical, and mechanical properties due to their exceptionally light weight, large surface area, and strong C-C bonds when compared with traditional materials[1-4]. Hence, CNT network-based materials have attracted widespread attention in the areas of technology research and manufacture. For example, assembled CNTs with well-ordered orientations and configurations, such as three-dimensional sponge-array architectures, CNT foams, and CNT aerogels, have demonstrated unprecedented mechanical and thermal properties compared with materials of similar density [5-9]. However, it is not easy to fabricate a well-ordered CNT network because of the likelihood of entanglement during the fabrication process $[10,11]$. Owing to the large van der Waals (vdW) interactions between carbon structures, CNTs are prone to aggregate and then form into bundles and entanglements, thus forming randomly distributed CNT networks which are collectively called 'buckypaper'[12-14]. Several mechanical and thermal tests for buckypapers have been implemented which indicate that buckypaper may be one of the most promising new nanomaterials [15-17]. For example, experimental results demonstrate that the Young's modulus of buckypaper can be tuned from 0.2GPa to $3.1 \mathrm{GPa}$ by varying the buckypaper's density and average CNT diameter $[18,19]$, while the Poisson's ratio can be tuned between positive and negative values by changing the content of multi-walled CNTs (MWCNTs) [14]. Remarkably, buckypapers normally possess extremely low densities of $0.05-0.4 \mathrm{~g} / \mathrm{cm}^{3}$ and high porosities of $0.8-0.9[20]$ which make buckypapers very flexible, and it has been shown that buckypaper composed of single-walled CNTs (SWCNTs) with diameters $0.8-1.2 \mathrm{~nm}$ and lengths $100-$ $1000 \mathrm{~nm}$ has a pore size of about $10 \mathrm{~nm}[21]$. 
In current research, buckypapers have been widely used in various applications, such as actuators, filtration systems, sensors, distillation devices, energy dissipation devices, etc.[22-31] For example, buckypaper-films composed of thin-walled carbon nanostructures filled with $\mathrm{Fe}_{3} \mathrm{C}$, FeCo, FeNi, Co, and Ni crystals can obtain enhanced saturation magnetization compared with pristine $\mathrm{Co}, \mathrm{CoNi}$ and $\mathrm{Ni}$ [23]. Chatterjee et al. [28] reported that buckypaper electrodes display superior sensitivity towards tryptophan, L-carnitine, tyrosine, and myoglobin; when engineered with specific molecules the buckypaper shows up to a 1000-fold increase in signal compared with electrodes based on glassy carbon. Rein et al. [31] found that buckypaper sensors are able to measure strains in polymer with different elastic properties while Chen et al. [29] revealed that buckypapers have outstanding impact behavior and high energy dissipation efficiency up to 95\% when subjected to impacts. Additionally, Liu et al. [32] found that the interlaminar fracture toughness of a composite laminate can be greatly improved by incorporating the buckypaper into the middle interface of the laminate. Basiuk et al. [33] demonstrated the possibility of fast and efficient solvent-free functionalization of buckypaper mats prefabricated from oxidized MWCNTs by utilizing three typical amines of different structure. Han et al. [34] made use of buckypaper-based coatings consisting of conductive buckypaper and insulating adhesives to protect carbon fiber reinforced polymer laminates. Ma et al. [35] put forward a flexible tactile and shear sensing array incorporating novel patterned buckypaper which exhibited favorable sensitivity and repeatability. Miao [36] revealed that alumina-filled hybrid buckypaper composites have strong interfacial polarization, high conductivity, and enhanced microwave absorbing performance.

Beyond these applications, it is also worth noting that several papers indicate that buckypapers exhibit frequency- and temperature-independent viscoelastic properties from $-196^{\circ}$ to $1000^{\circ} \mathrm{C}$ 
and that the zipping-unzipping mechanism during the loading-unloading process can result in strong energy dissipation [37, 38]. Moreover, Li et al. [37] also investigated the effects of double-walled CNT (DWCNT) content on viscoelasticity of buckypaper. Viscoelasticity features attributes of both viscosity and elasticity simultaneously, indicating capability towards energy dissipation and reversible deformation, which both play important roles in many biological and engineering systems, such as vibration abatement, injury prevention, spacecraft construction, satellite stability, gasket reliability, computer disk storage, tire performance, earplug functionality, and many others [39]. However, most current research pertinent to the mechanical properties of buckypaper focus on purely elastic rather than viscoelastic properties. Therefore, this work performs coarse-grained non-equilibrium molecular dynamic simulations to give further insight into the viscoelasticity of SWCNT buckypapers. We mainly focus on the effects

of oscillatory shear strain amplitude, buckypaper's density, and the length of incorporated SWCNTs on the viscoelastic properties of buckypapers. Our work aims to provide an in-depth understanding of the viscoelasticity of buckypaper relevant to its structure, and facilitate the design of new energy dissipation devices.

\section{Model and Computational Methods}

\subsection{Coarse-grained model for SWCNTs}

Since computational costs limit the implementation of fully atomistic molecular dynamics (MD) simulations for large CNT networks with a large range of vdW interactions, a coarse-grained molecular dynamics (CGMD) model for CNT networks is emerging as a complementary tool to access time and length scales the atomistic model inevitably cannot $[40,41]$. To this end, a fully atomistic CNT is discretized into multiple beads connected by elastic springs to form a chain model. The discrete beads interact through bonds between two adjacent beads, angles among 
three successive beads, and a pairwise potential between non-successive beads; this corresponds to stretching, bending, and intertubular adhesion energy respectively. The twisting behavior which may make a slight effect on the mechanical properties of buckypaper is often neglected, as this CG model with relevant force field has been proved by many publications to successfully describe the mechanical properties of the buckypaper, regardless of the incorporation of twisting behavior [42-44]. Therefore, the total energy of our model of the buckypaper can be expressed as:

$$
E_{\text {tot }}=E_{\text {bond }}+E_{\text {angle }}+E_{v d W}
$$

where the stretching energy is calculated as $E_{\text {bond }}=\sum_{\text {bonds }} \frac{1}{2} k_{b}\left(r-r_{0}\right)^{2} ; k_{b}$ being the stiffness constant, $r$ is the current bond length, and $r_{0}$ is the equilibrium bond length. The bending energy is given by $E_{\text {angle }}=\sum_{\text {angles }} \frac{1}{2} k_{a}\left(\theta-\theta_{0}\right)^{2}$, where $k_{a}$ is the bending constant, $\theta$ is the current angle and $\theta_{0}$ is the equilibrium angle. The vdW interaction is given by $E_{v d W}=$ $\sum_{\text {pairs }} 4 \varepsilon\left[(\sigma / r)^{12}-(\sigma / r)^{6}\right]$, where $\varepsilon$ denotes the depth of the potential well and $\sigma$ denotes the equilibrium distance where the inter-bead potential is zero. A relatively large cutoff of $28 \mathrm{~nm}$ is used for the vdW interactions, in order to more thoroughly capture the effects of entanglement and bundling between CNTs. Based on the equilibrium bond distance and the assumption of homogeneous distribution of CNT mass, the mass of each bead is determined to be $1,953.23 \mathrm{~g} /$ mol. The parameters $k_{b}, r_{0}, k_{a}, \theta_{0}, \varepsilon$, and $\sigma$ used in our CGMD simulations can be derived from the full atomistic MD simulations. Parameters $r_{0}, \theta_{0}$, and $\sigma$ can be obtained by equilibrium conditions while $k_{b}, k_{a}$, and $\varepsilon$ can be determined by energy conservation conditions based on tensile, bending, and adhesion tests. The details can be found in references $[19,45]$. In our simulations, the CGMD model of buckypaper is formed by $(5,5)$ SWCNTs (hereafter referred to as merely CNTs for short) and the potential parameters are presented in Table 1. This CGMD 
model of buckypaper using these parameters has been adopted in many publications, such as research into the behavior of entanglement and bundling, tensile tests, self-assembly, and so on $[19,40,42-44,46]$. The results of these computational studies have been proven to agree well with experimental results, which provides a firm foundation for our work by using the same CGMD model. It has to be noted that for any buckypaper in this work, all individual SWCNTs have the same length, avoiding the polydispersity effect. In order to investigate the effect of individual SWCNTs' length on the viscoelasticity of buckypapers, six models of SWCNTs with different lengths $(100 \mathrm{~nm}, 237 \mathrm{~nm}, 410 \mathrm{~nm}, 800 \mathrm{~nm}, 1896 \mathrm{~nm}$ and $6400 \mathrm{~nm})$ are used to construct six types of buckypapers respectively while for each buckypaper the sum length of all SWCNTs is kept at 51,200 $\mathrm{nm}$ by adjusting the number of SWCNTs. In addition, for the fixeddensity tests, the density of each specimen is set at $107.26 \mathrm{~kg} / \mathrm{m}^{3}$ which closely matches those reported by experiments $[14,22]$. We will now discuss how these SWCNT models are used to construct the buckypaper models.

\subsection{Configurations of buckypaper}

A random walk method combined with the excluded volume algorithm is adopted to generate the CGMD buckypaper model in order to mimic the randomness and isotropy of buckypaper. In the model generation process, a series of points are put into an orthogonal cell in a spatially uniform manner, serving as the positions for the initial bead of each SWCNT. By changing the cell size, buckypaper models with different densities can be obtained. It is obvious that the number of these initial points is equal to the number of SWCNTs in the specimen. Each SWCNT grows to the next position for the next bead by a random bond vector with the magnitude equal to the equilibrium bond length $(10 \AA)$. An excluded volume check is then performed to avoid the

overlapping of beads. The critical check distance is set to be $2 \AA$, that is to say, if the newly 
generated position is less than $2 \AA$ away from any other bead, it is considered that they are overlapped. On this occasion, the SWCNT returns back to the last position and finds a new position based on a new random bond vector, followed by a new overlap check. This findingchecking-returning process is repeated until the new position passes the overlap check. A SWCNT's walk terminates when the total number of steps is equal to the desired length of the SWCNT. It has to be noted that a partial overlap of the beads is likely to take place, which can be acceptable because these partially-overlap beads can reach their well-equilibrium positions after the subsequent energy minimization. The representative orthogonal simulation box is set to be periodic, to simulate a large, connected mass of buckypaper.

Following the model generation by random walk, an isothermal-isobaric (NPT) ensemble is used to relax the initial configuration of the buckypaper until the pressure and temperature of the system reach $1 \mathrm{~atm}$ and $300 \mathrm{~K}$, respectively. During the equilibrium process, the behavior of entanglement and aggregation of SWCNTs is observed due to the large range of $\mathrm{vdW}$ interactions, which is consistent with the conclusion reported by Li et al. [46] that entanglement behavior dominates the structure of SWCNT buckypaper while DWCNT buckypaper is mainly influenced by bundling behavior. The total energy of the buckypaper decreases rapidly and then approaches an equilibrium value slowly. In Figure 1, (a) and (b) depict snapshots of buckypaper models made with SWCNTs of individual lengths $100 \mathrm{~nm}$ and 6,400 nm, respectively. All coarse-grained molecular figures are represented with the visualization software OVITO [47].

\subsection{Non-equilibrium simulations of buckypaper}

The viscoelastic properties are investigated by applying an oscillatory shear deformation and recording the resulting stress. This is a non-equilibrium molecular dynamics (NEMD) problem due to an external field driving the buckypaper away from the equilibrium state. The SLLOD (so 
called because of its association with the DOLLS tensor algorithm[48]) equations of motion, one of the most widely used methods for NEMD problems, have been proven to be equivalent to Newton's equations of motion for shear flow [49] and are able to generate the desired velocity gradient and the correct production of work by stresses for all forms of homogeneous flow [50]. The models are coupled to a Nose-Hoover thermostat $[51,52]$ in a velocity Verlet formulation at a constant temperature of $300 \mathrm{~K}$ in our simulations. According to Tuckerman et al. [53], the SLLOD equations of motion can be expressed as :

$$
\begin{gathered}
\dot{\boldsymbol{q}}_{i}=\frac{\boldsymbol{p}_{i}}{m_{i}}+\boldsymbol{q}_{i} \cdot \boldsymbol{\nabla u}, \\
\dot{\boldsymbol{p}}_{i}=\boldsymbol{F}_{i}-\boldsymbol{p}_{i} \cdot \boldsymbol{\nabla u}-\frac{p_{\eta}}{Q} \boldsymbol{p}_{i}, \\
\dot{\eta}=\frac{p_{\eta}}{Q}, \\
\dot{p}_{\eta}=\sum_{i=1}^{N} \frac{\boldsymbol{p}_{i}^{2}}{m_{i}}-d N k_{B} T
\end{gathered}
$$

where, $\boldsymbol{q}_{i}, \boldsymbol{p}_{i}, \boldsymbol{F}_{i}$ and $m_{i}$ are the position vector, the momentum vector, the force vector and the mass of the $i-$ th bead, and $\boldsymbol{u}$ is the streaming velocity. The dot denotes a derivative with respect to time and $\boldsymbol{\nabla}$ is a spatial gradient. $\eta$ and $p_{\eta}$ are the coordinate- and momentum-like variables, respectively. $d$ is the dimension of space, $N$ is the total number of beads, $k_{B}$ is the Boltzmann constant and $T$ is the temperature of the system. $Q$ is the thermostat mass parameter expressed as $d N k_{B} T \tau^{2}$, where $\tau$ is the characteristic relaxation time and is equal to 1 ps in this work with a timestep of $10 \mathrm{fs}$. For the SLLOD method, Lees-Edwards periodic boundary conditions [54] for SLLOD equations are applied, which generate homogeneous isothermal flow. The orthogonal simulation box is changed to be non-orthogonal, in preparation for the shear tests. Coordinates and velocities of atoms are adjusted to create a shear velocity profile, so that each point in the simulation box has a streaming velocity. This position-dependent streaming velocity is 
subtracted from each atom's actual velocity to yield a thermal velocity for temperature computation and thermostatting. Velocities of atoms crossing the periodic boundaries are remapped, keeping consistent with the velocity profile. In this work, the simulation box is being sheared along the $x$ direction, relative to $y$. The shear strain is defined as $\gamma_{x y}=d_{x} / L_{y 0}$, where $d_{x}$ is the difference of transverse displacements between the upper and lower $x z$ planes, and $L_{y 0}$ is the box length in the $y$ direction and is a constant in this work. For the oscillatory shear simulations, the shear strain is given by a sinusoidal function:

$$
\gamma_{x y}=\gamma_{0} \sin (\omega t)
$$

where $\gamma_{0}$ and $\omega$ are the strain amplitude and angular frequency of the oscillatory shear deformation, respectively, and $t$ is the time. The angular velocity $\omega=2 \pi f$, where $f$ is the oscillatory shear frequency. In our simulations, in order to find the viscoelasticity as a function of the strain amplitude, the shear strain amplitude is varied from $0.01-0.5$ while the frequency is kept at $500 \mathrm{MHz}$. To find the relationship between the shear frequency and the viscoelasticity, the shear frequency is changed from $50-2,000 \mathrm{MHz}$ at constant strain amplitude 0.05 . Thus, the effects due to the length of individual CNTs on the viscoelasticity can be explored based on the variations of length and frequency at strain amplitude 0.05 . The simulations last for 30 cycles of oscillatory shear deformation and the averaged shear stress is derived by using the latter 15 cycles, with the first 15 cycles discounted in order to allow for settling time. According to the theory of linear viscoelasticity [55], the output shear stress for buckypaper also has a sinusoidal form with a phase shift relative to the input shear strain, expressed as:

$$
\tau_{x y}=\tau_{0} \sin (\omega t+\delta)
$$


where $\tau_{0}$ is the stress amplitude and $\delta$ is the phase angle. The stress during the latter 15 cycles of the shear deformation is recorded and then fitted using a sinusoidal function with the same frequency as the input strain, as depicted in Figure 2, where the R-square is around 0.997, showing an exceedingly close fit. The storage shear modulus $G^{\prime}$, loss shear modulus $G^{\prime \prime}$, and loss $\operatorname{tangent} \tan \delta$ which represent the linear viscoelastic properties of buckypaper are defined as:

$$
\begin{aligned}
& G^{\prime}=\left(\tau_{0} / \gamma_{0}\right) \cos \delta \\
& G^{\prime \prime}=\left(\tau_{0} / \gamma_{0}\right) \sin \delta \\
& \tan \delta=G^{\prime} / G^{\prime \prime}
\end{aligned}
$$

The loss angle $\delta$ is a dimensionless measure of the viscoelastic damping of the material. For a linearly elastic solid, $\delta=0$, and for a viscous fluid, $\delta=\pi / 2$. Thus for viscoelastic materials, $0<\delta<\pi / 2 . G^{\prime}$ is the component of the stress-strain ratio in phase with the applied strain, while $G^{\prime \prime}$ is the component $90^{\circ}$ out of phase. The single and double primes do not represent derivatives, but denote the real and imaginary parts respectively. The complex dynamic modulus can be obtained by $G^{*}=G^{\prime}+\mathrm{i} G^{\prime \prime}$ with the magnitude $\left|G^{*}\right|=\sqrt{\left(G^{\prime}\right)^{2}+\left(G^{\prime \prime}\right)^{2}}=\tau_{0} / \gamma_{0}$, where $\mathrm{i}=\sqrt{-1}$. Taking into account the stiffness and the energy dissipation simultaneously, a damping figure of merit $D$ describing the dynamic damping performance is defined as:

$$
D=\left|G^{*}\right| \tan \delta=\sqrt{1+\tan ^{2} \delta} G^{\prime \prime}
$$

Generally, for low damping materials the damping figure of merit $D$ is approximately equal to $G^{\prime \prime}$, ignoring the order of $\tan ^{2} \delta$. Here NEMD simulations of buckypaper are implemented to obtain $\tau_{0}$ and $\delta$, thus calculating $G^{\prime}, G^{\prime \prime}, \tan \delta$ and $D$ according to Eqs. (8) - (11).

\section{Results and Discussion}




\subsection{Strain amplitude effect}

In order to determine the range of the strain amplitude corresponding to the linear viscoelastic regime and its effect on the viscoelastic properties of buckypaper, NEMD simulations of buckypaper made with SWCNTs of individual length $100 \mathrm{~nm}$ are performed. The strain amplitude is varied over a wide range of 0.01-0.5 at a frequency of $500 \mathrm{MHz}$, covering noise, elastic, and plastic deformation regimes. The viscoelastic properties of buckypaper, including storage modulus $G^{\prime}$, loss modulus $G^{\prime \prime}$, damping figure of merit $D$, and loss factor $\tan \delta$, are presented in Figure 3. At extremely low strain amplitude $\left(\gamma_{0}<0.03\right)$, the shear storage modulus $G^{\prime}$ related to the elastic energy decreases so slightly that it can be considered to be strain amplitude insensitive. The reason is probably that $G^{\prime}$ is related to the elastic energy, and so during the elastic regime it remains stable. Contrarily $G^{\prime \prime}$, related to the dissipated energy, increases from $1.17 \mathrm{MPa}$ to $5.33 \mathrm{MPa}$ and $\tan \delta$ increases from 0.04 to 0.24 when strain amplitude grows from 0.01 to 0.03 , which means that the viscosity increases quickly during this regime. This is probably related to the noise effect resulting from thermal fluctuations. As the strain amplitude grows, the noise effect becomes softer. In addition, since the length of the simulation box is about $1,100 \mathrm{~nm}$, when the strain varies from 0.01 to 0.03 the displacement of the upper surface (Figure 4) ranges from $11 \mathrm{~nm}$ to $33 \mathrm{~nm}$, which is just close to the distance $10 \mathrm{~nm}$ between adjacent bonded atoms. Thus, the deformation during the oscillatory shear tests is rather tiny, especially for strain amplitude 0.01 , and so the zipping-unzipping behavior occurs in few entanglement sites, which results in very little viscosity ( $\operatorname{small} \tan \delta$ and $G^{\prime \prime}$ ). As the strain amplitude grows, more and more entanglement sites are able to display the zippingunzipping mechanism, enhancing the viscosity (larger $\tan \delta$ and $G^{\prime \prime}$ ). Since in this regime $\tan \delta$ is small, the damping figure $D=\left|G^{*}\right| \tan \delta=\sqrt{1+\tan ^{2} \delta} G^{\prime \prime}$ is approximately equal to $G^{\prime \prime}$. As 
is observed in Figure 3, for $0.03 \leq \gamma_{0} \leq 0.05$, all the values of the viscoelastic properties $\left(G^{\prime}\right.$, $G^{\prime \prime}, D$, and $\tan \delta$ ) remain nearly independent of the shear strain amplitude $\gamma_{0}$, indicating a linear response for buckypaper subjected to external oscillatory shear deformation. Likewise, experimental results showed that the maximum strain amplitude for the reversible deformation linear regime is 0.05 [38]. Past the linear regime, the buckypaper shows nonlinear viscoelastic properties due to plastic deformation of the buckypaper. Thus as the strain amplitude increases, the phase angle increases in an approximately linear fashion; however, the shear storage modulus $G^{\prime}$ decreases due to a decrease in the elastic stiffness. The loss modulus $G^{\prime \prime}$ remains nearly constant for $0.05<\gamma_{0}<0.2$, indicating invariance in energy dissipation. As the strain amplitude continues to increase, the damping ratio still rises rather linearly while both the storage modulus $G^{\prime}$ and the loss modulus $G^{\prime \prime}$ decrease, exhibiting a process of temporary shear thinning at moderate strains [56]. The difference between $G^{\prime}$ and $G^{\prime \prime}$ becomes smaller until $\gamma_{0}=$ 0.5 where $G^{\prime} \approx G^{\prime \prime}$. After that, $G^{\prime \prime}$ begins to be larger than $G^{\prime}$. The variation tendency between $G^{\prime}$ and $G^{\prime \prime}$ agrees well with relevant experimental results [38]. It also can be found that beyond the critical strain amplitude $\left(\gamma_{0}=0.05\right)$ the damping figure of merit $D$ gradually becomes noticeably different from the loss modulus, thanks to the increment of the damping ratio.

Figure 4 depicts snapshots of the buckypaper model at three representative states: undeformed state $\left(\gamma_{x y}=0\right)$, reversible deformation $\left(\gamma_{x y}=0.05\right)$, and highly irreversible deformation $\left(\gamma_{x y}=\right.$ 0.5). At the undeformed state, the buckypaper exhibits a 3D highway network where each SWCNT is in contact with many other SWCNTs. Unlike the bundle dominated buckypaper where numerous CNTs are nearly straight and contact with each other over a long distance, the entanglement behavior of long CNTs makes the buckypaper possess high porosity. This porous nature of the buckypaper is favorable for fast and efficient heat dissipation, avoiding 
considerable heat accumulation. In addition, the entanglement of SWCNTs yields a large range of reversible deformation, and thus sustains large linear strain amplitudes up to 0.05 . The connections between SWCNTs, also called entanglement sites, are usually over a short span. Attachment and detachment between SWCNTs occur reversibly during the cyclic oscillatory deformation by zipping and unzipping mechanisms respectively, which is also observed by both experiment [38] and simulation [37]. As Figure 5 shows, during the loading process (a)-(c), zipping behavior between two SWCNTs occurs, forming an entanglement. Unzipping behavior then happens during the unloading process, reducing the inter-tube entanglements. Plenty of energy is consumed during the unzipping process in order to overcome the large vdW attraction between SWCNTs, yet the zipping process is spontaneous. Thus due to the high density of entanglement sites the buckypaper is believed to have excellent energy dissipation capability. Beyond the linear regime, several SWCNTs tend to align and become bundled, which begins to take shape in Figure 4(c) and will be more obvious under larger strain according to the experimental results [38]. Therefore the number of entanglement sites is reduced, resulting in a degeneration of the energy dissipation capability.

In the linear regime, the output stress is a typical sinusoidal function versus time, seen in Figure 6(a) where the strain amplitude $\gamma_{0}=0.05$ is small. Another characteristic of the linear viscoelasticity is the so called elliptical Lissajous plot (Figure 6(c)), where the shear stress is plotted versus the input shear strain within a cycle of oscillation. This loop demonstrates a hysteresis phenomenon that is a lag between cause and effect. The area of the loop denotes the energy dissipation density (energy dissipation per unit volume per cycle of the material) which is proportional to the viscosity modulus $G^{\prime \prime}$. In the nonlinear regime, the time-dependent stress deviates from the sinusoidal function resulting in a distortion of the elliptical loop, which can be 
seen in Figure 6(b) and (d) where the strain amplitude $\gamma_{0}=0.5$, leading to high nonlinearity. Thus, a Fourier-transform method can be employed in order to describe the stress distortion by calculating high odd harmonics contribution, which can be expressed in the sine and cosine form[57]:

$$
\tau_{x y} / \gamma_{0}=\sum_{k=1,3,5, \ldots}\left[G_{k}^{\prime} \sin (k \omega t)+G_{k}^{\prime \prime} \cos (k \omega t)\right]
$$

where $k$ is odd. It has been proven that the Fourier expansion can contain only odd modes since

clockwise and anticlockwise rotations are mechanically equivalent, and thus only odd values of $k$ are used. Figure 6(b) and (d) exhibit fitting by Eq. (11) where only the first two odd modes $(k=1,3)$ are necessary for a near-perfect fitting with R-square up to 0.99 . It is obvious that the Fourier-transform method is also applicable in the linear regime using only the first mode, which can be expressed as:

$$
\tau_{x y} / \gamma_{0}=G^{\prime} \sin (\omega t)+G^{\prime \prime} \cos (\omega t)
$$

The following discussions in this paper are based on the strain amplitude $\gamma_{0}=0.05$ to ensure that the buckypaper remains within the linear regime while still having a significant oscillation.

\subsection{Density effects}

Density, as one of the primary parameters to determine the structure of buckypaper, has been proven to strongly influence mechanical properties such as the Young's modulus, bulk modulus, impact behavior, etc.[20, 29] Thus it is meaningful to discuss the effects of density on the viscoelastic properties of buckypaper. Because the energy dissipation of buckypaper is dominated by the zipping-unzipping mechanism at entanglement sites, we adopt the $Z 1$ code [58-61], a purely geometric algorithm, to count the number of entanglement sites of the 
buckypaper system, a parameter which is difficult to measure in experiments. In the $Z 1$ code, each buckypaper system is mapped on a multiple disconnected path of straight lines which is infinitesimally thin, impenetrable, and tensionless. The primitive path made of a linear chain with several kinks and segments connects the space-fixed ends of a CNT and is minimized for a shortest path while maintaining the underlying entanglement structure. Subjected to lineuncrossability, the total length of all the lines for a buckypaper system is reduced by introducing fewer nodes. To acquire a shortest multiple disconnected path carrying entanglement information, nodes which do not change the direction of the path are removed; two segments without overlap with other obstacles become a single one, otherwise they will change their orientation simultaneously; two segments may grow to three if more than one obstacle hinders the straightening behavior. The number of final internal nodes (interior kinks) represents the number of entanglements of the buckypaper system. This $Z 1$ code has been applied by researchers to networks such as polymeric [60,62, 63] and buckypaper-based [46] systems for acquiring entanglement structure and entanglement analysis. Therefore, using this $Z 1$ code the number of inter-tube entanglements per CNT for a buckypaper, denoted as $Z_{e}$, versus density of buckypaper is displayed in Figure 7(a).

Figure 7(a) depicts that for lower densities (from $39.7 \mathrm{~kg} / \mathrm{m}^{3}$ to $49.4 \mathrm{~kg} / \mathrm{m}^{3}$ ) $Z_{e}$ grows little with increasing density. Yet for larger densities (from $61.6 \mathrm{~kg} / \mathrm{m}^{3}$ to $107.3 \mathrm{~kg} / \mathrm{m}^{3}$ ), $Z_{e}$ increases approximately linearly and the trend climbs as the density of the buckypaper rises. The viscoelastic properties of buckypaper as a function of density can be found in Figure 7(b). As the density grows, both the loss factor and loss modulus keep increasing and the increment rate tends to increase as well, indicating higher energy dissipation capability at higher densities, due to the larger $Z_{e}$. It can be deduced that the energy dissipation is not simply proportional to $Z_{e}$, but they 
have a close relationship since $Z_{e}$ strongly dominates the energy dissipation capability. The buckypaper storage modulus also increases at higher densities due to the stronger elastic stiffness which has been demonstrated in our previous work [29]. The density varying from $39.70 \mathrm{~kg} / \mathrm{m}^{3}$ to $107.30 \mathrm{~kg} / \mathrm{m}^{3}$ results in an increase of $4.2,6.8$, and 1.6 times for the storage modulus, loss modulus, and damping ratio, respectively. Similarly, experimental results also show that when the density of buckypaper increases 4 times, the increments of storage and loss modulus and the damping ratio are 5, 10 and 2 times respectively[38].

\subsection{Length effects}

The length of individual CNTs is another important factor in determining the structure of buckypaper. However, there are no current studies in its effects on the viscoelastic properties of buckypaper. Experiments about frequency-independence showed constant viscoelastic properties including storage modulus, loss modulus, and damping ratio of the buckypaper in the range of 0.1 to $100 \mathrm{HZ}$ [38]. Related simulations also proved the frequency-independence of the viscoelasticity of buckypaper for the range between $15.7 \mathrm{MHz}$ and $3 \mathrm{GHz}$ [37], but for significantly higher frequencies the viscoelasticity becomes frequency-dependent. Since most engineering applications rarely involve extremely high frequencies, in this segment we focus on the frequency-independent regime and the effects of SWCNT length on viscoelastic properties within this frequency-independent regime. Our results in Figure 8 exhibit frequency-independent viscoelasticity for the range of $50 \mathrm{MHz}$ to $1 \mathrm{GHz}$. It is known that the energy dissipation capability of buckypaper mainly depends on the zipping-unzipping mechanism between SWCNTs, which is a fast process. If the shear loading rate is below the zipping-unzipping rate, energy dissipation during a cycle is believed to be rate- or frequency-independent. It has to be noted that the critical frequencies ending the frequency-independence regime are not the same 
for all samples because of the variant internal structures and manufacture processes of buckypaper. If the loading rate is beyond the zipping-unzipping rate, the hysteresis between the input strain and output stress becomes more severe, resulting in a larger damping ratio $\tan \delta$ and thus a higher loss modulus. The shear storage modulus decreases, indicating smaller dynamic elastic stiffness.

It is also shown in Figure 8 that the individual length of SWCNTs has noticeable influence on the viscoelastic properties. For oscillatory shear frequency $f \leq 1 \mathrm{GHz}$, the damping ratio $\tan \delta$ of a buckypaper remains independent of the length of individual SWCNTs over a wide range from $100 \mathrm{~nm}$ to $6400 \mathrm{~nm}$, indicating an invariance of the ratio of energy dissipated to energy stored in dynamic loading. In this regime, as the length of individual SWCNTs rises, the loss modulus exhibits a slight decline and seems to reach a plateau corresponding to a critically large length of individual SWCNTs, which can be seen in Figure 9(b). This can be further understood by the $Z 1$ code analysis shown in Figure 9(a). The number of inter-tube entanglements per SWCNT $\left(Z_{e}\right)$ increases approximately linearly with increasing length of individual SWCNTs, which can be expressed as $Z_{e}=k L_{C N T}+b$ ( $k$ is the slope, $b$ is a positive constant and $L_{C N T}$ is the length per CNT). The total number of inter-tube entanglements in a buckypaper system can be obtained by $Z_{t}=N \times Z_{e} / 2$ ( $N$ is the number of chains), as each entanglement involves two SWCNTs. Since $N=N_{t} / L_{C N T}\left(N_{t}=51,200\right.$, is the total number of beads in each buckypaper system), we can get $Z_{t}\left(L_{C N T}\right)=\left(b N_{t} / L_{C N T}+k N_{t}\right) / 2=46,976 / L_{C N T}+31,155$, and thus $d Z_{T} / d L_{C N T}=$ $-46,976 / L_{C N T}^{2}$. Therefore, for the length of individual SWCNTs $\left(L_{C N T}\right)$ varying from $100 \mathrm{~nm}$ to $6400 \mathrm{~nm}$, the total number of entanglements in a buckypaper system $Z_{t}\left(L_{C N T}\right)$ slightly decreases with a reducing rate, and becomes stable with relatively longer SWCNTs, which is coincident with the variation trend of the loss modulus. With the growth of the length of 
individual CNTs, the storage modulus also decreases, and the decline gradually appears to reach a plateau. The reason is probably that SWCNTs with larger lengths are more prevalent to bending and buckling when deformed, resulting in a more flexible buckypaper and thus a lower stiffness. This buckling phenomenon was clearly observed in early experiments and MD simulations and it was reported that the CNTs have remarkable buckling capacity and that the buckled CNTs have the ability to fully recover elastically [64-67] corresponding to the linear regime of viscoelasticity. Cao and Chen [68] found that the critical bending curvature grows with respect to the aspect ratio, and after the aspect ratio reaches a critical value the critical buckling curvature becomes insensitive to the aspect ratio. Similarly, after the length is beyond a critical value $(400 \mathrm{~nm})$, the storage modulus associated with elastic energy becomes unchanged with respect to SWCNT length, which is depicted in Figure 9(b). It has to be noted that, strictly speaking, the loss modulus and damping figure of merit have slight variation with respect to the SWCNT length, but this dependence is so slight that they can be considered to be approximately independent of SWCNT length.

For $f$ beyond the critical value $(1 \mathrm{GHz})$, the damping ratio of buckypaper seems to be more sensitive to the length of individual SWCNTs, as is seen in Figure 8. The reason is probably that a buckypaper made of longer SWCNTs has more bending and buckling deformation, resulting in a larger lag between the input strain and output stress subjected to the oscillatory shear deformation with significantly high frequency. That is to say, buckypaper made of longer SWCNTs behaves more like a viscoelastic material than buckypaper made of shorter SWCNTs does. Figures S1 and S2 in the Supporting Information illustrate the trend of viscoelasticity between $1 \mathrm{GHz}$ and $2.3 \mathrm{GHz}$ in detail. It can be found that for CNT lengths from $100 \mathrm{~nm}$ to $1896 \mathrm{~nm}$, shear storage modulus $G^{\prime}$ declines slightly while shear loss modulus $G^{\prime \prime}$, loss factor 
$\tan \delta$, and damping figure of merit $D$ increase slightly with respect to CNT length. For CNT length of $6400 \mathrm{~nm}$, there appears something different: as frequency varies from $1.9 \mathrm{GHz}$ to $2.3 \mathrm{GHz}, \tan \delta$ and $D$ increase quickly. That is related to the increase of phase angle $\delta$. After $\delta$ reaches $\sim 1 \mathrm{rad}$, a slight increase of $\delta$ can result in an extreme increase of $\tan \delta$ and thus a rapid increase of $D$, which may result in the calculation error for the loss factor and damping figure of merit. Here, we mainly focus on the frequency-independent regime, and thus the corresponding effects and properties of buckypaper at high frequencies $(>1 \mathrm{GHz})$ need to be further investigated in the future.

Figure 10 depicts the elliptic Lissajous figures for different lengths of individual SWCNTs under oscillatory shear frequencies $500 \mathrm{MHz}$ and $2 \mathrm{GHz}$, representing frequency-independence and frequency-dependence respectively. The values of storage and loss moduli can be revealed in the elliptic Lissajous figure directly and the width of the figure is a measure of the loss angle $\delta$. The enclosed area of the elliptic Lissajous figure represents the dissipated energy per volume of the material during a period, which is presented in Figure 11. It is obvious that the dissipated energy per volume per cycle with respect to the length of individual SWCNTs under different oscillatory shear frequencies has a similar variation trend as the loss storage modulus does. In the regime of frequency-independence, with the increase of SWCNT length the dissipated energy declines slightly from around $0.04 \mathrm{~J} / \mathrm{cm}^{3}$ to around $0.03 \mathrm{~J} / \mathrm{cm}^{3}$. Therefore, the dissipated energy can be considered to be approximately independent of SWCNT length. For $f=2 \mathrm{GHz}$, energy dissipation is obviously higher at lower frequencies.

\section{Conclusions}


In this paper, a series of coarse-grained NEMD simulations of buckypaper with $(5,5)$ SWCNTs subjected to oscillatory shear deformation are performed in order to investigate the effects of oscillatory shear strain amplitude and frequency, buckypaper density, and length of the constituent SWCNTs on the viscoelastic properties of buckypaper. The key findings revealed by our simulation results are as follows: (i) with the growth of the strain amplitude the viscoelasticity of buckypaper experiences noise, linear, and nonlinear regimes in turn, and the linear regime in this paper corresponds to a strain amplitude range of $0.03 \leq \gamma_{0} \leq 0.05$; the damping ratio for the linear regime is around 0.25 which is much higher than those of conventional materials ( $\sim 10^{-3}$ or less for metals). (ii) Energy dissipation is mainly dominated by the zipping-unzipping mechanism at entanglement sites; the loss modulus related to energy dissipation is not simply proportional to the number of entanglements (which can be calculated by $Z 1$ code), however they do have a close relationship. (iii) Due to an increase in entanglements and the resulting stronger stiffness of buckypapers with higher density, the loss factor, loss modulus, storage modulus, and damping figure of merit all increase with increasing density, and the increment rate becomes larger for larger densities. (iv) Buckypapers exhibit frequencyindependent viscoelasticity when the frequency is below a critical value $(1 \mathrm{GHz})$; for frequencies beyond the critical value, both damping ratio and loss modulus increase indicating larger energy dissipation while the storage modulus decreases indicating smaller elastic energy. (v) In the frequency-independent regime, the shear storage modulus decreases slightly with respect to the length of individual SWCNTs; the damping ratio, the loss modulus and damping figure of merit are approximately independent of the length of SWCNTs, which may be attributed to the linear increasing of $Z_{e}$ with respect to the length. It has to be noted that buckypaper used in this work is made of pure $(5,5)$ SWCNTs; MWCNT buckypapers have potentially different properties. 
Overall, this work can provide deeper insight into the viscoelasticity of buckypaper and thus benefit the design of novel energy-dissipation devices for protecting critical structures or humans under extreme conditions by using fabricated carbon nanomaterials.

\section{Acknowledgements}

HC, JC, and HN appreciate the support from the Scholarship of Nanjing University of Aeronautics and Astronautics (China). LZ, MB, and XW would like to acknowledge support from the University of Georgia (UGA) Research Foundation. Calculations are performed at the UGA Advanced Computing Resource Centre.

\section{Author information}

Corresponding author: Xianqiao Wang; Hong Nie

Email: xqwang@uga.edu; hnie@nuaa.edu.cn .

Competing financial interests: The authors declare no competing financial interests. 


\section{References}

[1] Chen X, Huang Y. Nanomechanics modeling and simulation of carbon nanotubes. Journal of Engineering Mechanics. 2008;134(3):211-6.

[2] Delfani MR, Shodja HM, Ojaghnezhad F. Mechanics and morphology of single-walled carbon nanotubes: from graphene to the elastica. Philosophical Magazine. 2013;93(17):2057-88. [3] Salvetat JP, Bonard JM, Thomson NH, Kulik AJ, Forró L, Benoit W, et al. Mechanical properties of carbon nanotubes. Appl Phys A. 1999;69(3):255-60.

[4] Chen H, Zhang L, Becton MD, Nie H, Chen J, Wang X. Molecular Dynamic Study of a CNT/Buckyballs-enabled Energy Absorption System. Physical Chemistry Chemical Physics. 2015;17:17311-21.

[5] Gui X, Zeng Z, Zhu Y, Li H, Lin Z, Gan Q, et al. Three-Dimensional Carbon Nanotube Sponge-Array Architectures with High Energy Dissipation. Advanced Materials. 2014;26(8):1248-53.

[6] Lattanzi L, De Nardo L, Raney JR, Daraio C. Geometry-Induced Mechanical Properties of Carbon Nanotube Foams. Advanced Engineering Materials. 2014;16(8):1026-31.

[7] Liu LQ, Ma WJ, Zhang Z. Macroscopic Carbon Nanotube Assemblies: Preparation, Properties, and Potential Applications. Small. 2011;7(11):1504-20.

[8] Bryning MB, Milkie DE, Islam MF, Hough LA, Kikkawa JM, Yodh AG. Carbon nanotube aerogels. Advanced Materials. 2007;19(5):661-4.

[9] Kim KH, Vural M, Islam MF. Single-walled carbon nanotube aerogel-based elastic conductors. Advanced materials. 2011;23(25):2865-9.

[10] Ma W, Song L, Yang R, Zhang T, Zhao Y, Sun L, et al. Directly synthesized strong, highly conducting, transparent single-walled carbon nanotube films. Nano Letters. 2007;7(8):2307-11.

[11] Dai H, Kong J, Zhou C, Franklin N, Tombler T, Cassell A, et al. Controlled chemical routes to nanotube architectures, physics, and devices. The Journal of Physical Chemistry B. 1999;103(51):11246-55.

[12] Rinzler A, Liu J, Dai H, Nikolaev P, Huffman C, Rodriguez-Macias F, et al. Large-scale purification of single-wall carbon nanotubes: process, product, and characterization. Applied Physics A: Materials Science \& Processing. 1998;67(1):29-37.

[13] Thess A, Lee R, Nikolaev P, Dai H, Petit P, Robert J, et al. Crystalline ropes of metallic carbon nanotubes. Science-AAAS-Weekly Paper Edition. 1996;273(5274):483-7.

[14] Hall LJ, Coluci VR, Galvao DS, Kozlov ME, Zhang M, Dantas SO, et al. Sign change of Poisson's ratio for carbon nanotube sheets. Science. 2008;320(5875):504-7.

[15] Romo-Herrera JM, Terrones M, Terrones H, Meunier V. Guiding electrical current in nanotube circuits using structural defects: a step forward in nanoelectronics. ACS nano. 2008;2(12):2585-91.

[16] Li Y, Qiu X, Yang F, Yin Y, Fan Q. Stretching-dominated deformation mechanism in a super square carbon nanotube network. Carbon. 2009;47(3):812-9.

[17] Zsoldos I, Laszlo I. Computation of the loading diagram and the tensile strength of carbon nanotube networks. Carbon. 2009;47(5):1327-34.

[18] Zhang L, Zhang G, Liu C, Fan S. High-Density Carbon Nanotube Buckypapers with Superior Transport and Mechanical Properties. Nano Letters. 2012;12(9):4848-52.

[19] Cranford SW. In silico assembly and nanomechanical characterization of carbon nanotube buckypaper. Nanotechnology. 2010;21(26):265706. 
[20] Whitby RLD, Fukuda T, Maekawa T, James SL, Mikhalovsky SV. Geometric control and tuneable pore size distribution of buckypaper and buckydiscs. Carbon. 2008;46(6):949-56.

[21] Wu Q, Zhu W, Zhang C, Liang Z, Wang B. Study of fire retardant behavior of carbon nanotube membranes and carbon nanofiber paper in carbon fiber reinforced epoxy composites. Carbon. 2010;48(6):1799-806.

[22] Sears K, Dumee L, Schutz J, She M, Huynh C, Hawkins S, et al. Recent Developments in Carbon Nanotube Membranes for Water Purification and Gas Separation. Materials. 2010;3(1):127-49.

[23] Guo J, Lan M, Wang SL, He Y, Zhang SJ, Xiang G, et al. Enhanced saturation magnetization in buckypaper-films of thin walled carbon nanostructures filled with $\mathrm{Fe} 3 \mathrm{C}$, FeCo, FeNi, CoNi, Co and Ni crystals: the key role of Cl. Physical Chemistry Chemical Physics. 2015;17(27):18159-66.

[24] Cao Q, Kim H-s, Pimparkar N, Kulkarni JP, Wang C, Shim M, et al. Medium-scale carbon nanotube thin-film integrated circuits on flexible plastic substrates. Nature.

2008;454(7203):495-500.

[25] Cao Q, Rogers JA. Ultrathin Films of Single-Walled Carbon Nanotubes for Electronics and Sensors: A Review of Fundamental and Applied Aspects. Advanced Materials. 2009;21(1):29-53.

[26] Brady-Estévez AS, Kang S, Elimelech M. A Single-Walled-Carbon-Nanotube Filter for Removal of Viral and Bacterial Pathogens. Small. 2008;4(4):481-4.

[27] Meng C, Liu C, Fan S. Flexible carbon nanotube/polyaniline paper-like films and their enhanced electrochemical properties. Electrochemistry Communications. 2009;11(1):186-9.

[28] Chatterjee J, Cardenal J, Shellikeri A. Engineered Carbon Nanotube Buckypaper: A Platform for Electrochemical Biosensors. J Biomed Nanotechnol. 2015;11(1):150-6.

[29] Chen H, Zhang L, Chen J, Becton M, Wang X, Nie H. Energy dissipation capability and impact response of carbon nanotube buckypaper: A coarse-grained molecular dynamics study. Carbon. 2016;103:242-54.

[30] Chen IWP, Liang Z, Wang B, Zhang C. Charge-induced asymmetrical displacement of an aligned carbon nanotube buckypaper actuator. Carbon. 2010;48(4):1064-9.

[31] Rein MD, Breuer O, Wagner HD. Sensors and sensitivity: Carbon nanotube buckypaper films as strain sensing devices. Composites Science and Technology. 2011;71(3):373-81.

[32] Liu L, Shen LL, Zhou YX. Improving the interlaminar fracture toughness of carbon/epoxy laminates by directly incorporating with porous carbon nanotube buckypaper. $\mathrm{J}$ Reinf Plast Compos. 2016;35(2):165-76.

[33] Basiuk EV, Ramirez-Calera IJ, Meza-Laguna V, Abarca-Morales E, Perez-Rey LA, Re $\mathrm{M}$, et al. Solvent-free functionalization of carbon nanotube buckypaper with amines. Appl Surf Sci. 2015;357:1355-68.

[34] Han JH, Zhang H, Chen MJ, Wang D, Liu Q, Wu QL, et al. The combination of carbon nanotube buckypaper and insulating adhesive for lightning strike protection of the carbon fiber/epoxy laminates. Carbon. 2015;94:101-13.

[35] Ma C-W, Hsu L-S, Kuo J-C, Yang Y-J. A flexible tactile and shear sensing array fabricated using a novel buckypaper patterning technique. Sensors and Actuators a-Physical. 2015;231:21-7.

[36] Miao HY, Liu JH, Saravanan L. Fabrication of alumina filled hybrid buckypaper composites and their enhancement of microwave absorbing performance. J Phys D-Appl Phys. 2015;48(21):6. 
[37] Li Y, Kroeger M. Viscoelasticity of carbon nanotube buckypaper: zipping-unzipping mechanism and entanglement effects. Soft Matter. 2012;8(30):7822-30.

[38] Xu M, Futaba DN, Yamada T, Yumura M, Hata K. Carbon nanotubes with temperatureinvariant viscoelasticity from-196 to 1000 C. Science. 2010;330(6009):1364-8.

[39] Lakes RS. Viscoelastic materials: Cambridge University Press; 2009.

[40] Buehler MJ. Mesoscale modeling of mechanics of carbon nanotubes: Self-assembly, selffolding, and fracture. Journal of Materials Research. 2006;21(11):2855-69.

[41] Cranford S, Yao H, Ortiz C, Buehler MJ. A single degree of freedom 'lollipop' model for carbon nanotube bundle formation. Journal of the Mechanics and Physics of Solids.

2010;58(3):409-27.

[42] Yang X, He P, Gao H. Modeling frequency-and temperature-invariant dissipative behaviors of randomly entangled carbon nanotube networks under cyclic loading. Nano Research. 2011;4(12):1191-8.

[43] Wang C, Wang L, Xu Z. Enhanced mechanical properties of carbon nanotube networks by mobile and discrete binders. Carbon. 2013;64:237-44.

[44] Li Y, Kröger M. Computational study on entanglement length and pore size of carbon nanotube buckypaper. Applied Physics Letters. 2012;100(2):021907.

[45] Cranford S, Buehler MJ. Mechanomutable carbon nanotube arrays. International Journal of Materials and Structural Integrity. 2009;3(2-3):161-78.

[46] Li Y, Kroeger M. A theoretical evaluation of the effects of carbon nanotube entanglement and bundling on the structural and mechanical properties of buckypaper. Carbon.

2012;50(5):1793-806.

[47] Stukowski A. Visualization and analysis of atomistic simulation data with OVITO-the Open Visualization Tool. Modelling and Simulation in Materials Science and Engineering. 2010;18(1):015012.

[48] Morriss GP, Evans DJ. Statistical Mechanics of Nonequilbrium Liquids: ANU Press; 2007.

[49] Evans DJ, Morriss G. Nonlinear-response theory for steady planar Couette flow. Physical Review A. 1984;30(3):1528.

[50] Daivis PJ, Todd B. A simple, direct derivation and proof of the validity of the SLLOD equations of motion for generalized homogeneous flows. The Journal of chemical physics. 2006;124(19):194103.

[51] Nosé S. A unified formulation of the constant temperature molecular dynamics methods. The Journal of Chemical Physics. 1984;81(1):511-9.

[52] Hoover WG. Canonical dynamics: Equilibrium phase-space distributions. Physical Review A. 1985;31(3):1695-7.

[53] Tuckerman ME, Mundy CJ, Balasubramanian S, Klein ML. Modified nonequilibrium molecular dynamics for fluid flows with energy conservation. The Journal of Chemical Physics. 1997;106(13):5615-21.

[54] Lees A, Edwards S. The computer study of transport processes under extreme conditions. Journal of Physics C: Solid State Physics. 1972;5(15):1921-9.

[55] Ferry JD. Viscoelastic properties of polymers: John Wiley \& Sons; 1980.

[56] Renou F, Stellbrink J, Petekidis G. Yielding processes in a colloidal glass of soft star-like micelles under large amplitude oscillatory shear (LAOS). Journal of Rheology. 2010;54(6):121942. 
[57] Danov KD, Radulova GM, Kralchevsky PA, Golemanov K, Stoyanov SD. Surface shear rheology of hydrophobin adsorption layers: laws of viscoelastic behaviour with applications to long-term foam stability. Faraday discussions. 2012;158(1):195-221.

[58] Kröger M. Shortest multiple disconnected path for the analysis of entanglements in twoand three-dimensional polymeric systems. Computer Physics Communications. 2005;168(3):209-32.

[59] Shanbhag S, Kröger M. Primitive path networks generated by annealing and geometrical methods: insights into differences. Macromolecules. 2007;40(8):2897-903.

[60] Hoy RS, Foteinopoulou K, Kröger M. Topological analysis of polymeric melts: Chainlength effects and fast-converging estimators for entanglement length. Physical Review E. 2009;80(3):031803.

[61] Karayiannis NC, Kröger M. Combined molecular algorithms for the generation, equilibration and topological analysis of entangled polymers: Methodology and performance. International journal of molecular sciences. 2009;10(11):5054-89.

[62] Kim JM, Keffer DJ, Kröger M, Edwards BJ. Rheological and entanglement characteristics of linear-chain polyethylene liquids in planar Couette and planar elongational flows. Journal of Non-Newtonian Fluid Mechanics. 2008;152(1-3):168-83.

[63] Foteinopoulou K, Karayiannis NC, Laso M, Kröger M, Mansfield ML. Universal Scaling, Entanglements, and Knots of Model Chain Molecules. Phys Rev Lett. 2008;101(26):265702.

[64] Iijima S, Brabec C, Maiti A, Bernholc J. Structural flexibility of carbon nanotubes. The Journal of chemical physics. 1996;104(5):2089-92.

[65] Falvo M, Clary G, Taylor R, Chi V, Brooks F, Washburn S, et al. Bending and buckling of carbon nanotubes under large strain. Nature. 1997;389(6651):582-4.

[66] Knechtel WH, Düsberg GS, Blau WJ, Hernández E, Rubio A. Reversible bending of carbon nanotubes using a transmission electron microscope. Applied physics letters. 1998;73(14):1961-3.

[67] Lourie O, Cox D, Wagner H. Buckling and collapse of embedded carbon nanotubes. Phys Rev Lett. 1998;81(8):1638-41.

[68] Cao G, Chen X. Buckling of single-walled carbon nanotubes upon bending: Molecular dynamics simulations and finite element method. Physical Review B. 2006;73(15):155435. 
Table 1: Potential parameters for the coarse-grained model of $(5,5)$ SWCNT

\begin{tabular}{lc}
\hline Parameters & $(5,5)$ SWCNT \\
\hline Equilibrium bead distance, $\boldsymbol{r}_{\mathbf{0}}(\AA)$ & 10 \\
Stretching constant, $\boldsymbol{k}_{\boldsymbol{b}}\left(\mathbf{k c a l} /\left(\mathbf{m o l} \cdot \AA^{2}\right)\right)$ & 1,000 \\
Equilibrium angle, $\boldsymbol{\theta}_{\mathbf{0}}\left(^{\circ}\right)$ & 180 \\
Bending constant, $\boldsymbol{k}_{\boldsymbol{a}}\left(\mathbf{k c a l} /\left(\mathbf{m o l} \cdot \mathbf{r a d}^{2}\right)\right)$ & 14,300 \\
$\operatorname{vdW}$ distance, $\boldsymbol{\sigma}(\AA)$ & 9.35 \\
$\operatorname{vdW}$ energy, $\boldsymbol{\varepsilon}(\mathbf{k c a l} / \mathbf{m o l})$ & 15.10 \\
\hline
\end{tabular}



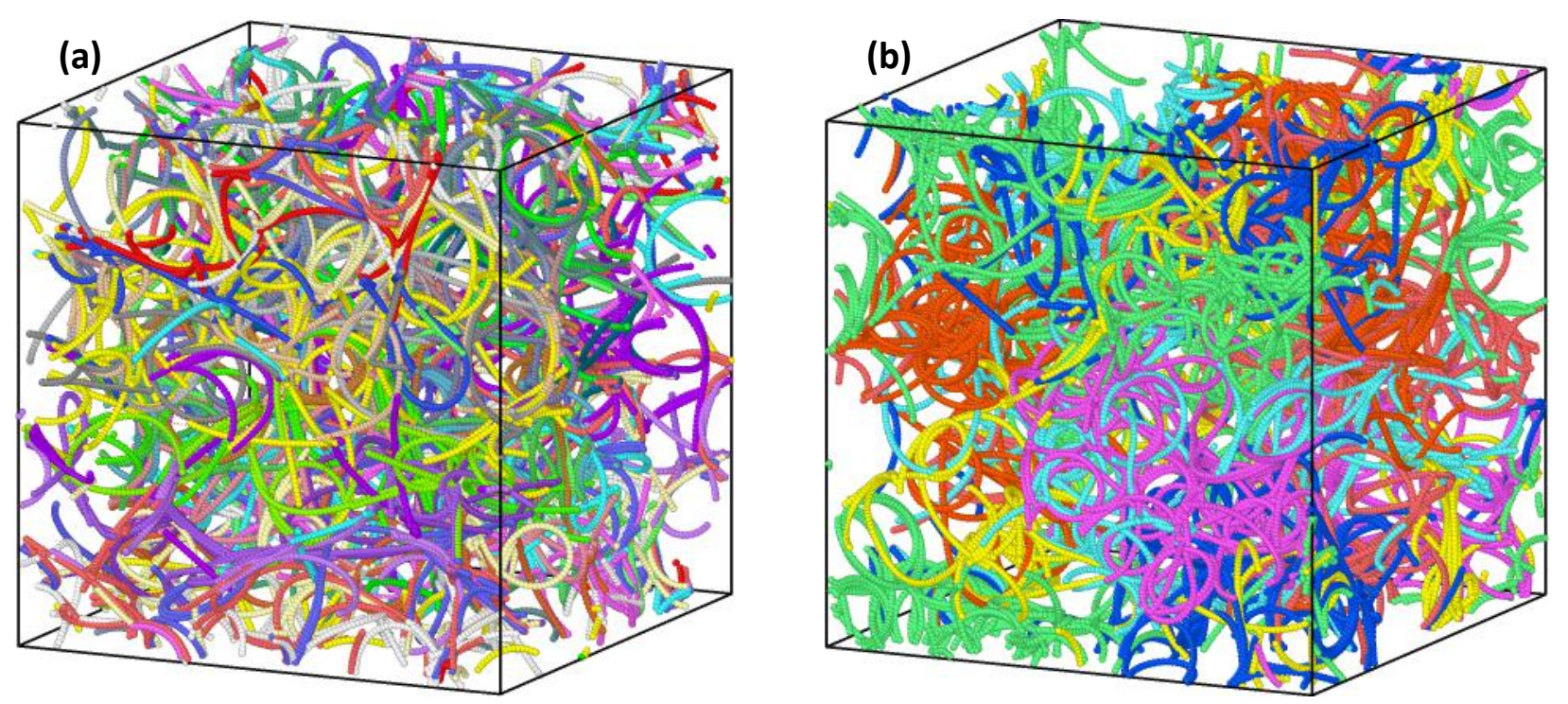

Figure 1: Computational cells of buckypapers made of CNTs with individual lengths (a) $100 \mathrm{~nm}$ and (b) 6, $400 \mathrm{~nm}$ at the equilibrium state. Both the total length of all the CNTs and the density of the buckypaper are kept the same. 

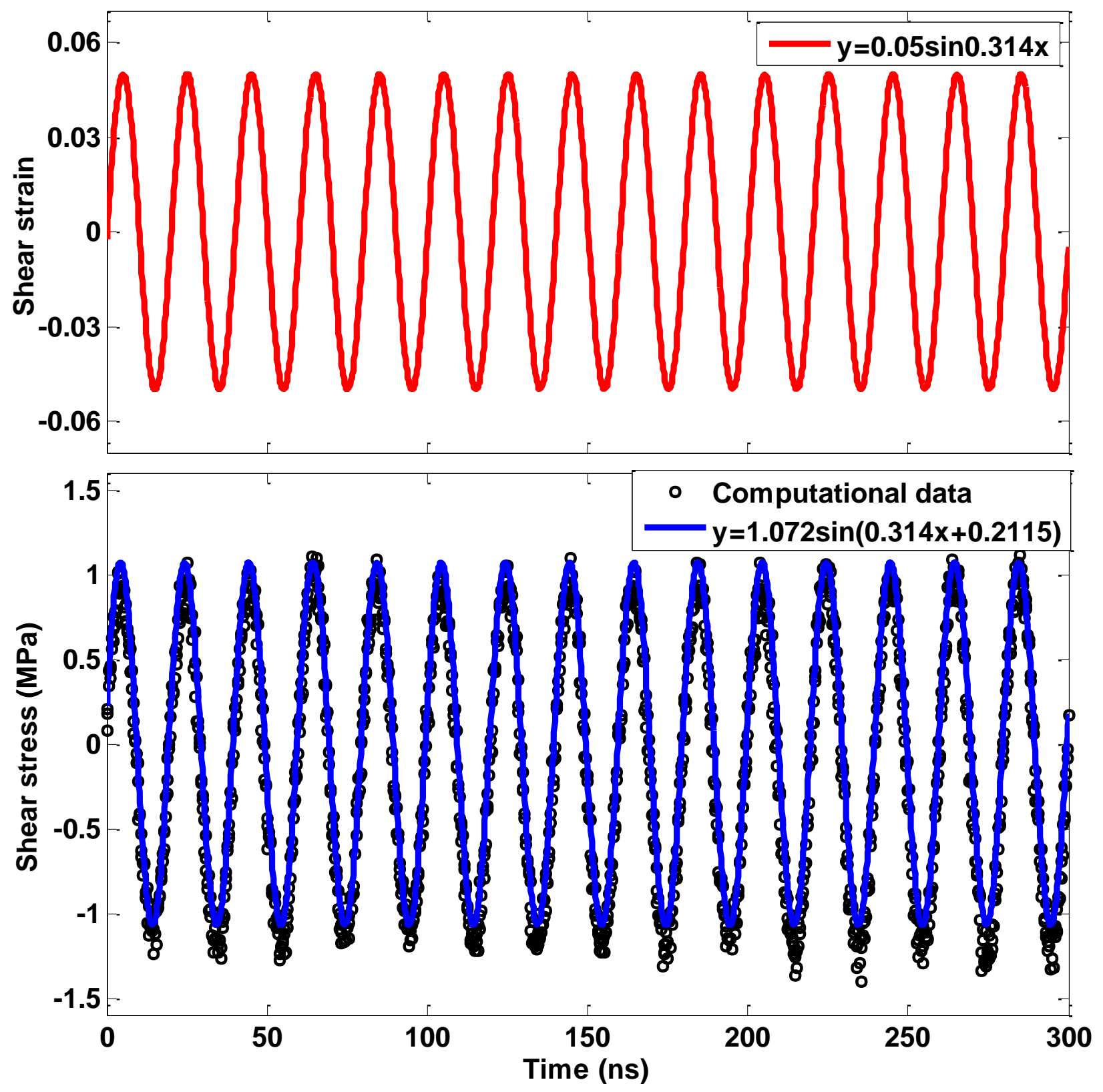

Figure 2: Input shear strain and output shear stress for the buckypaper made of CNTs with individual length $100 \mathbf{~ n m}$. The empty dots denote the computational shear stress and the continuous lines are sinusoidal fits to the empty dots. 


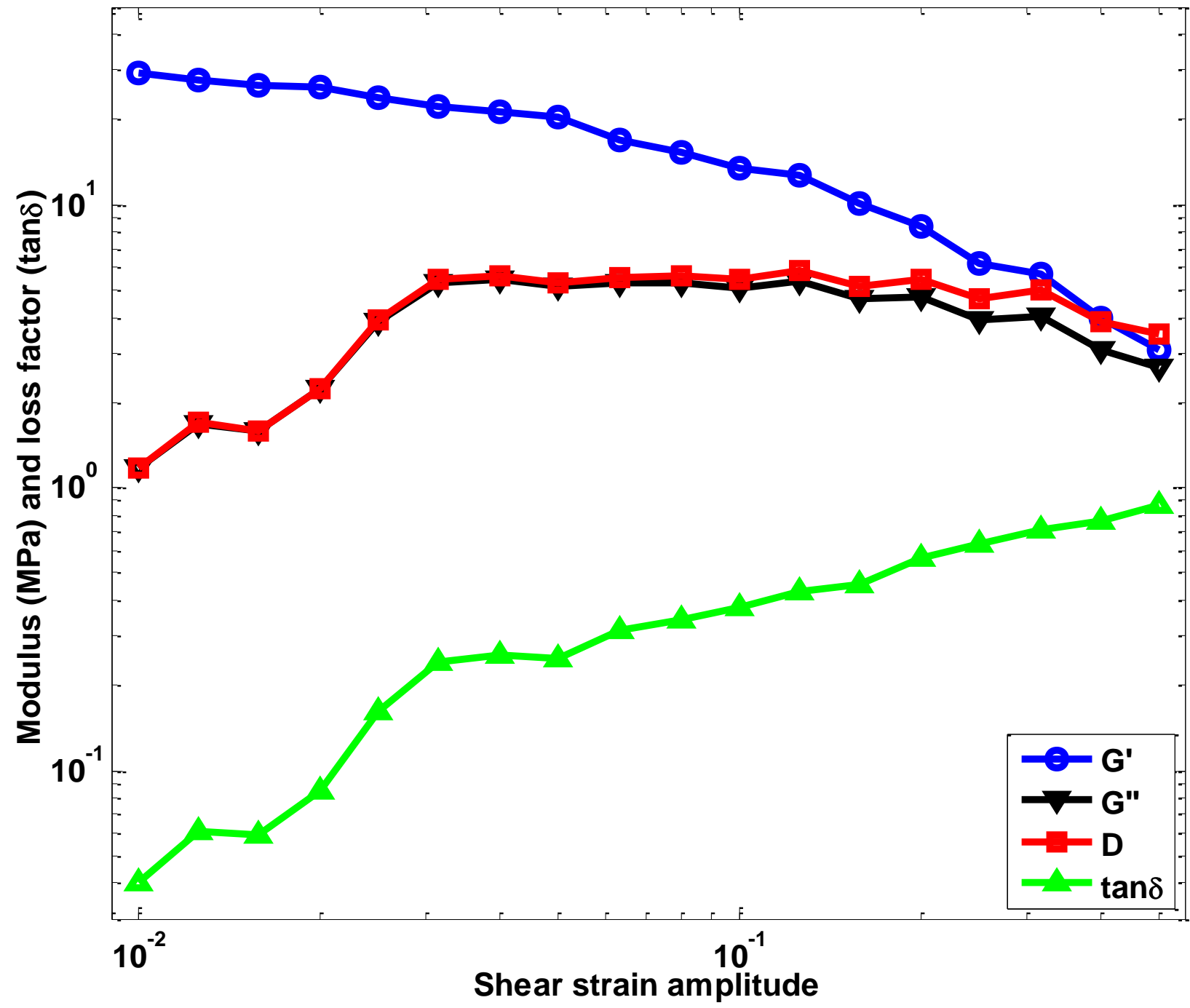

Figure 3: Shear storage modulus, loss modulus, damping figure of merit, and loss factor for buckypaper made of CNTs with individual length $\mathbf{1 0 0} \mathbf{~ n m}$ as a function of strain amplitude $(0.01-0.5)$. Shear frequency is $500 \mathrm{MHz}$. 


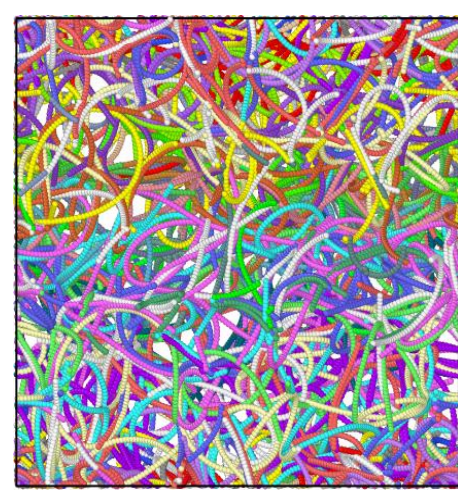

$$
\gamma_{x y}=0
$$

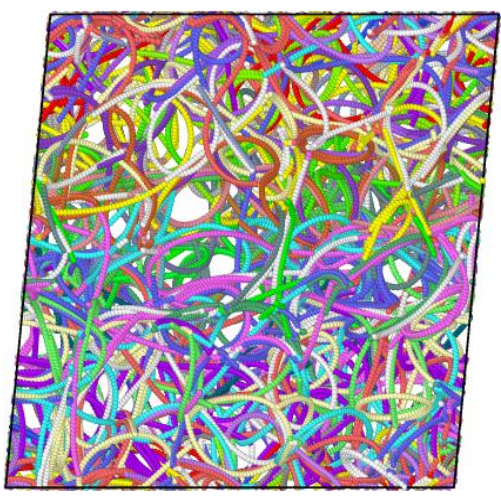

$\gamma_{x y}=0.05$

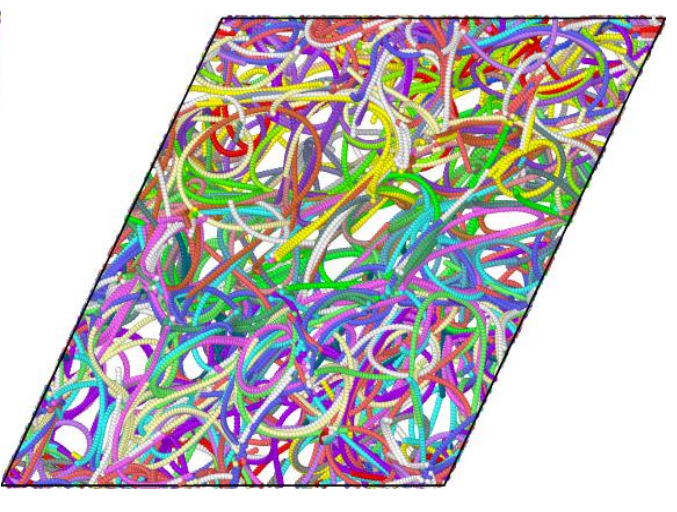

$\gamma_{x y}=0.5$

Figure 4: Snapshots of buckypaper made of 100 CNTs of individual length $\mathbf{1 0 0} \mathbf{~ n m}$ at varying shear strains. 


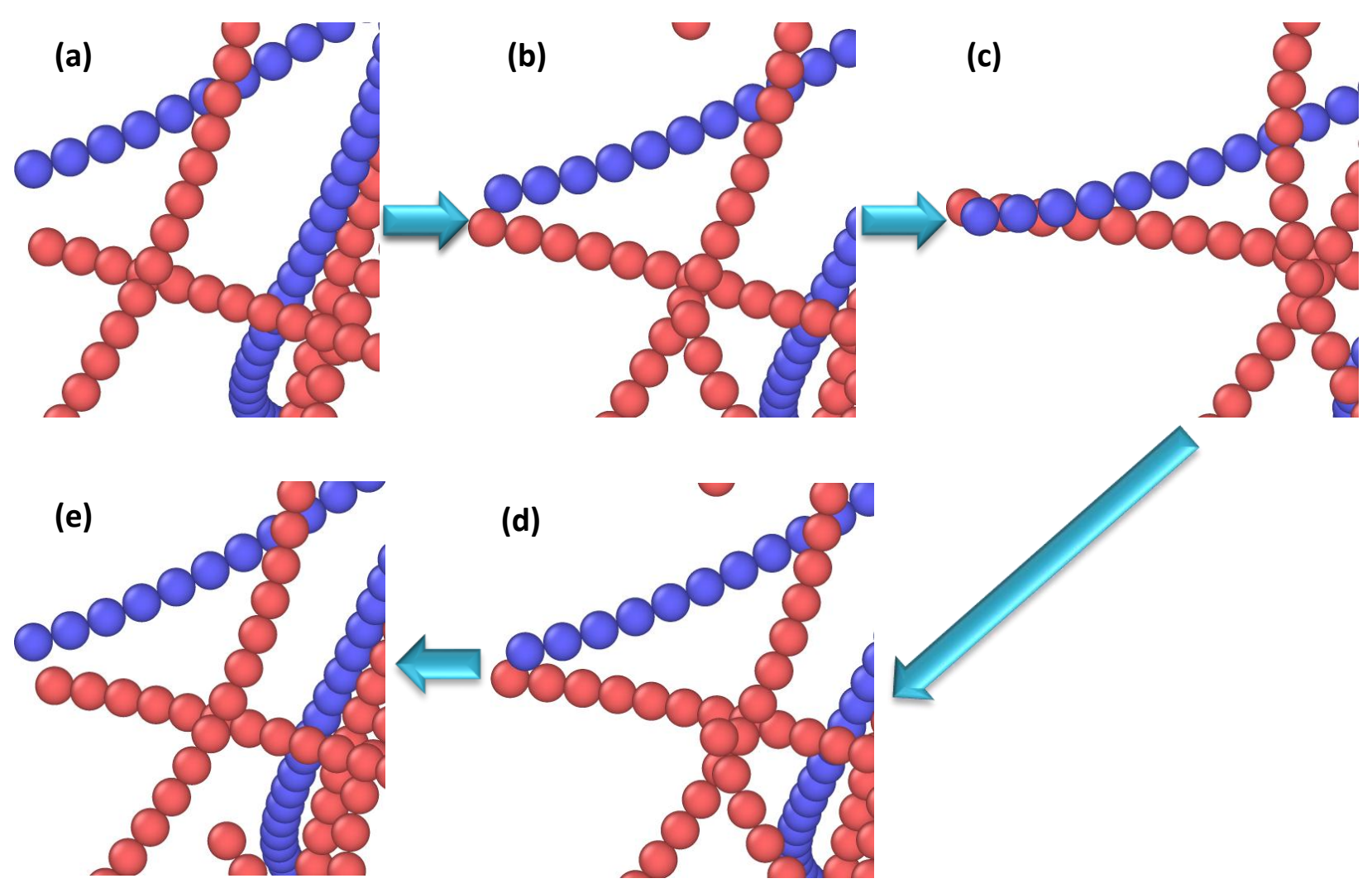

Figure 5: Snapshots of buckypaper made of 512 CNTs of individual length $\mathbf{1 0 0} \mathbf{~ n m}$ during a cyclic oscillatory shear deformation at a frequency of $\mathbf{5 0 0} \mathbf{~ M H z}$ and strain amplitude of 0.05 . Only several CNTs are selected for observation while others remain hidden. Different CNTs are colored differently. (a) demonstrates the original state; (a)-(c) depict the loading process during which zipping behavior of CNTs is observed; (c)-(e) show the unloading process when unzipping behavior of CNTs happens. 

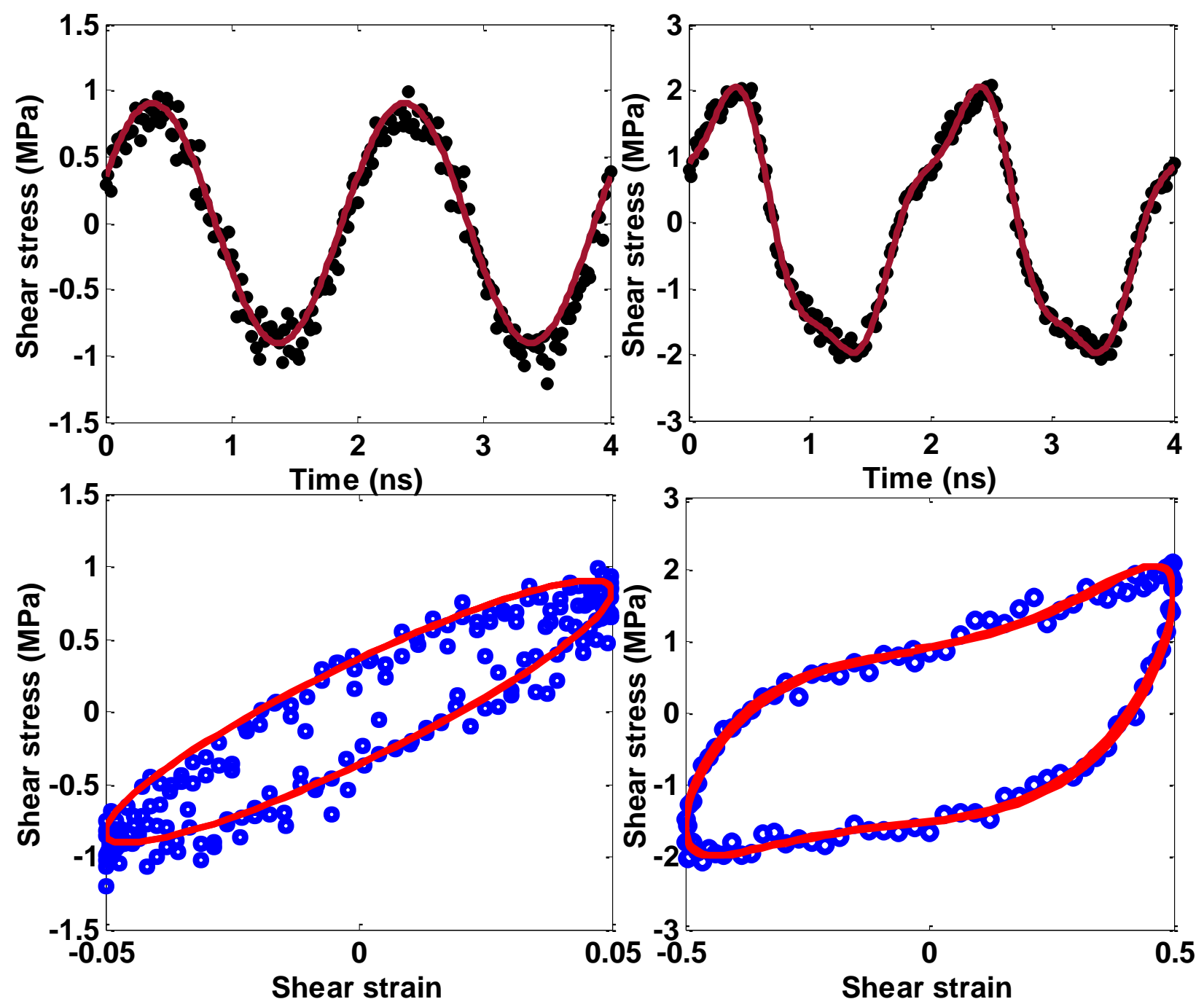

Figure 6: Shear stress response as a function of time for strain amplitude $\boldsymbol{\gamma}_{\mathbf{0}}=\mathbf{0 . 0 5}$ (a) and $\gamma_{\mathbf{0}}=\mathbf{0 . 5}$ (b) respectively. Lissajous plots of the shear stress versus the shear strain are shown for shear strain amplitudes $\gamma_{0}=\mathbf{0 . 0 5}$ (c) and $\gamma_{0}=\mathbf{0 . 5}$ (d) respectively. The dots denote the simulation data; the solid line in the left column represents the sinusoidal fit; the solid lines in the right column are fitted by a Fourier function. 

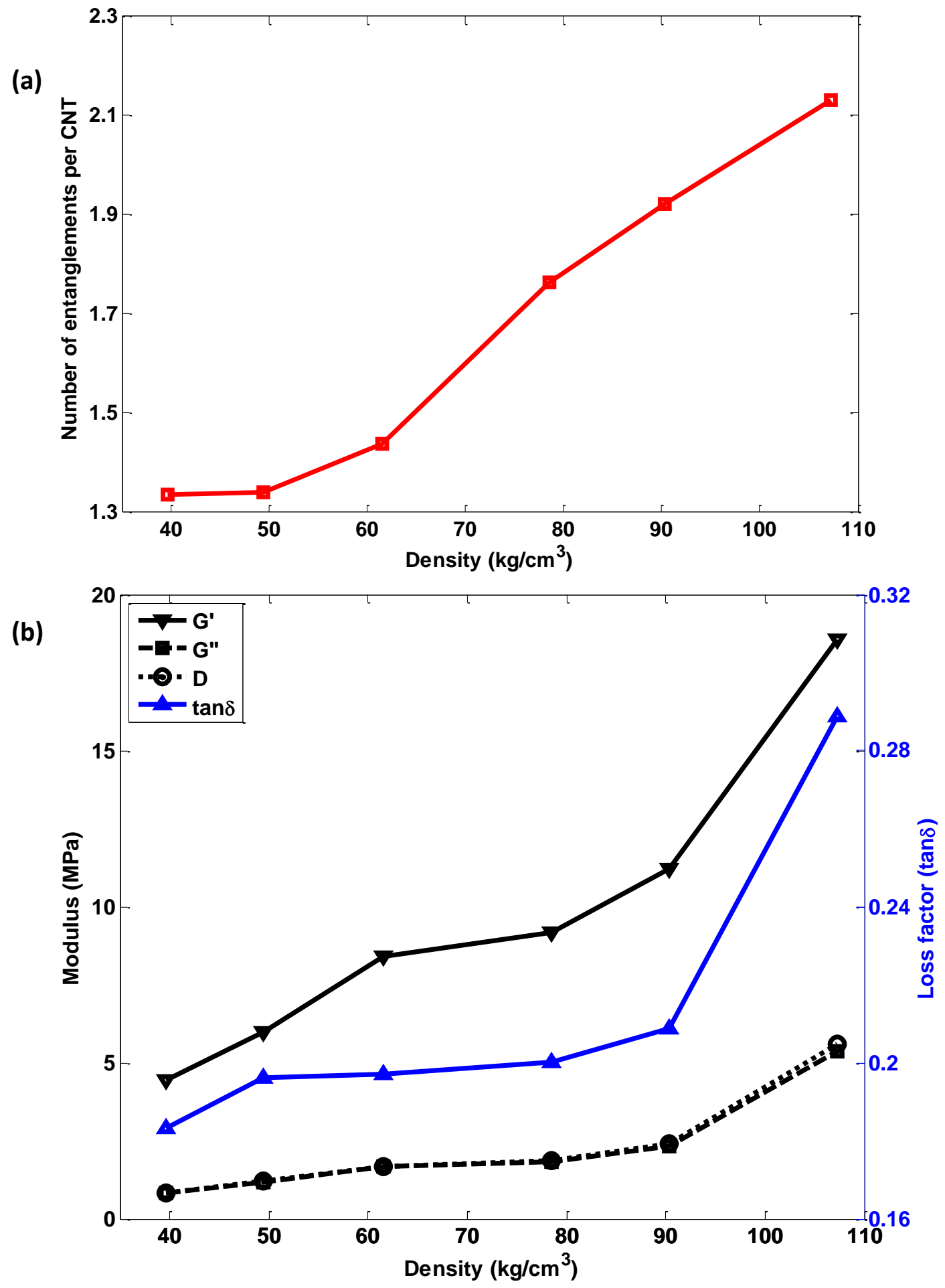

Figure 7: Number of entanglements per CNT (a) and viscoelastic properties (b) of buckypaper made of CNTs with individual length $100 \mathbf{~ n m}$ as a function of buckypaper's density. $\boldsymbol{\gamma}_{\mathbf{0}}=$ 0.05 and $f=500$ MPa. 


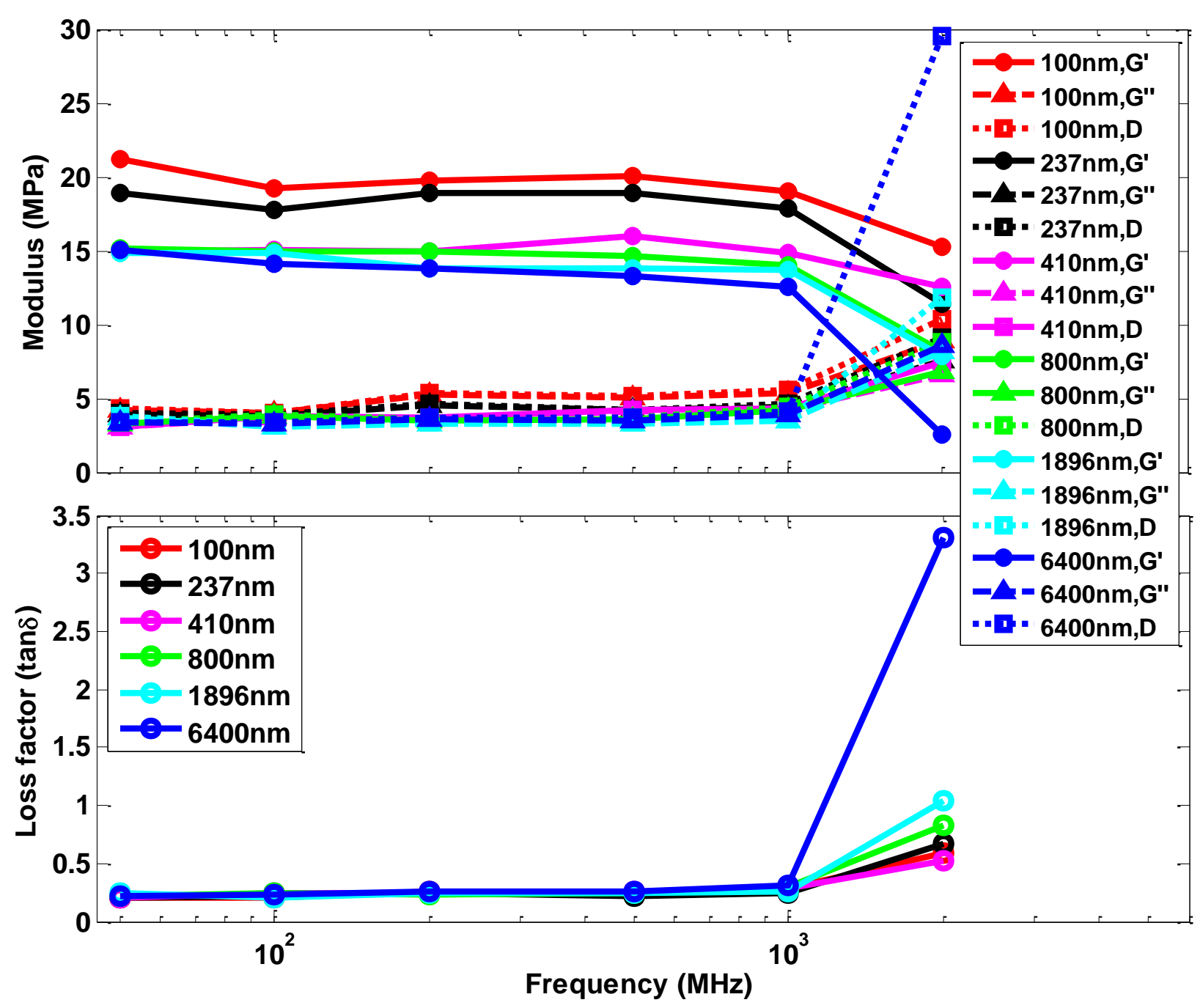

Figure 8: Shear storage modulus, loss modulus, damping figure of merit, and loss factor for buckypaper models made of CNTs with individual lengths from $100 \mathbf{n m}$ to $6400 \mathbf{n m}$ as a function of frequency $(50 \mathrm{MHz}-2000 \mathrm{MHz})$. 


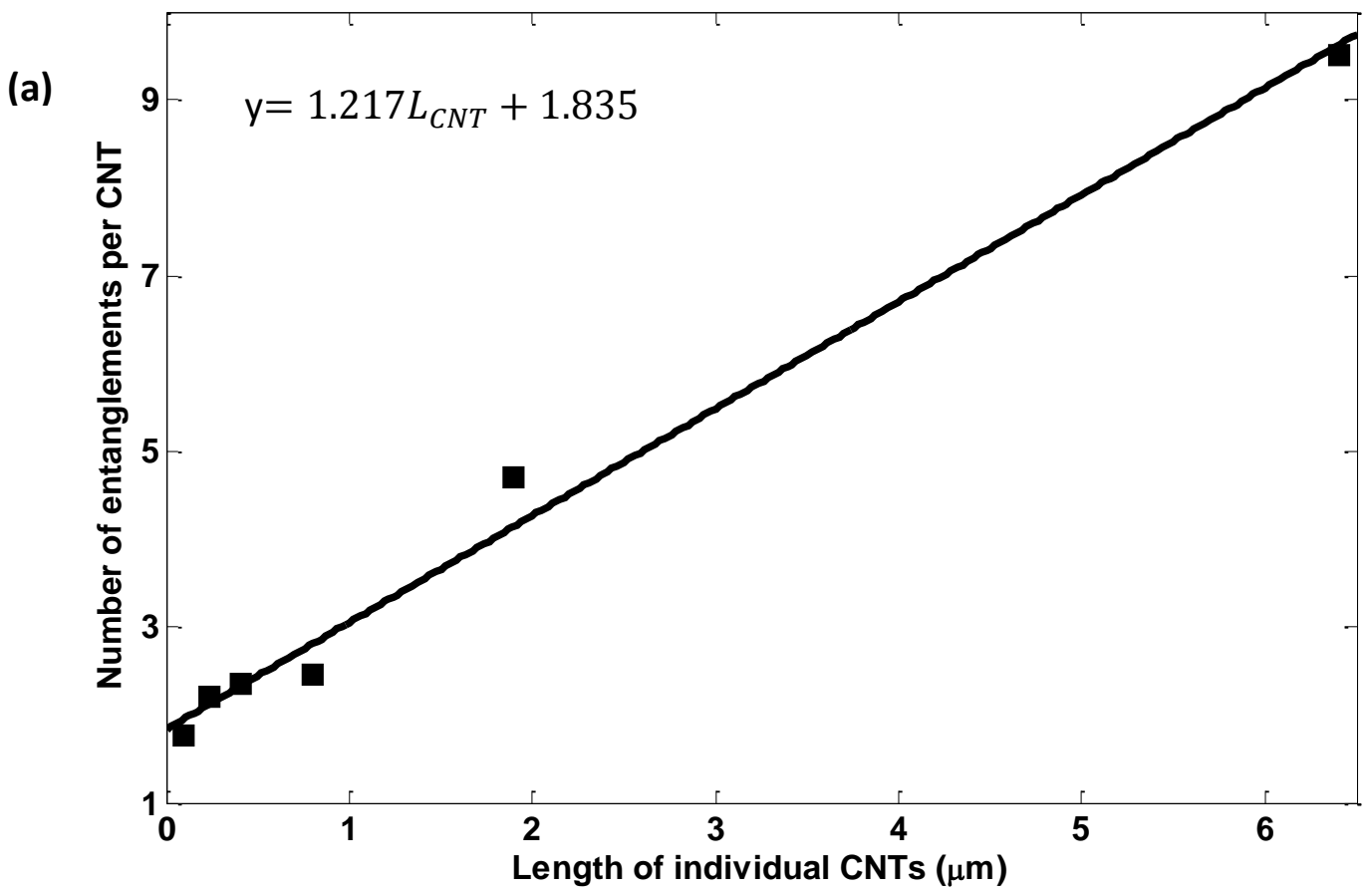

(b)

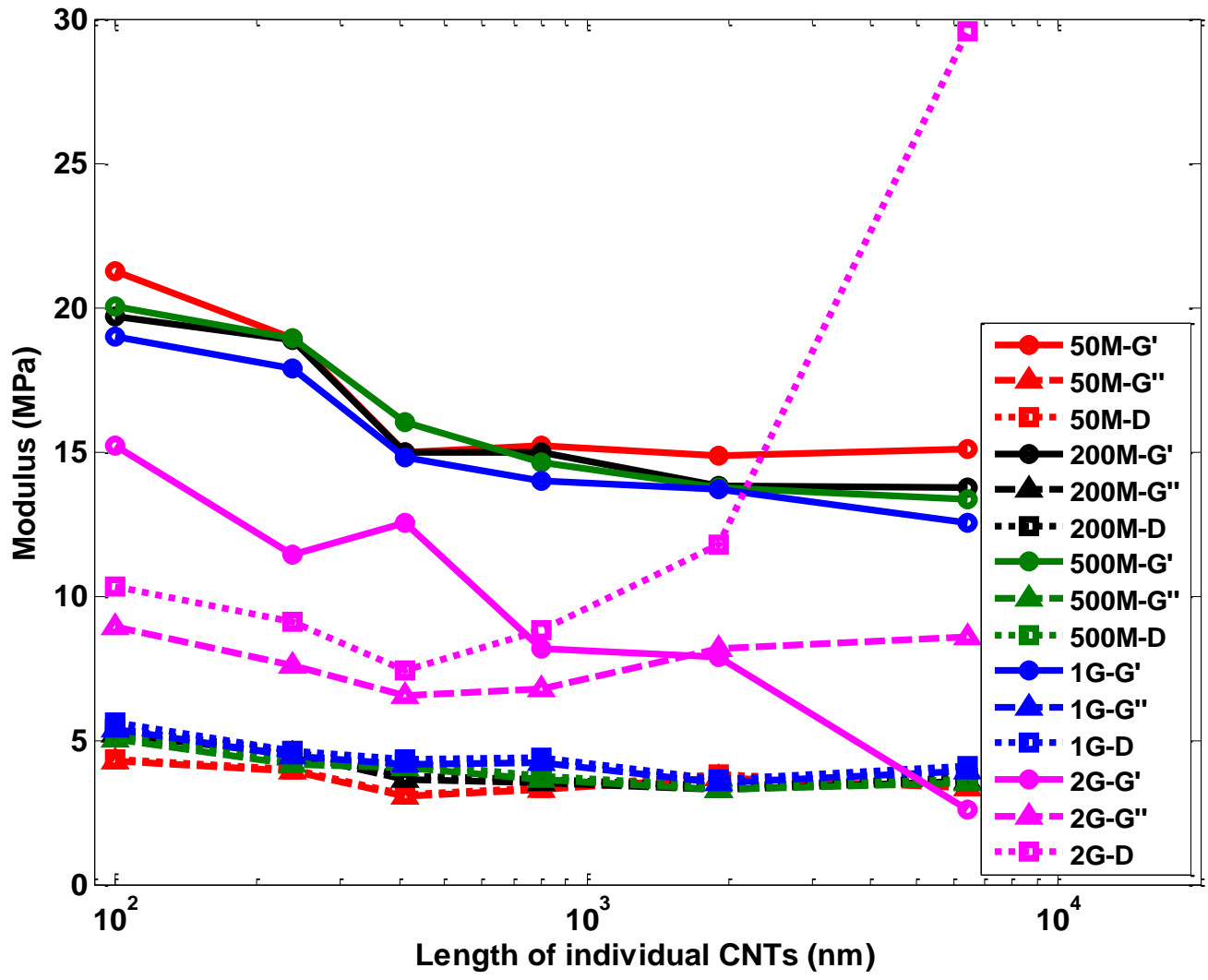

Figure 9: Number of entanglements per CNT (a) and viscoelastic properties of buckypaper (b) as a function of the length of individual CNTs from $100 \mathbf{n m}$ to $6400 \mathbf{n m}$ under different shear frequencies. 

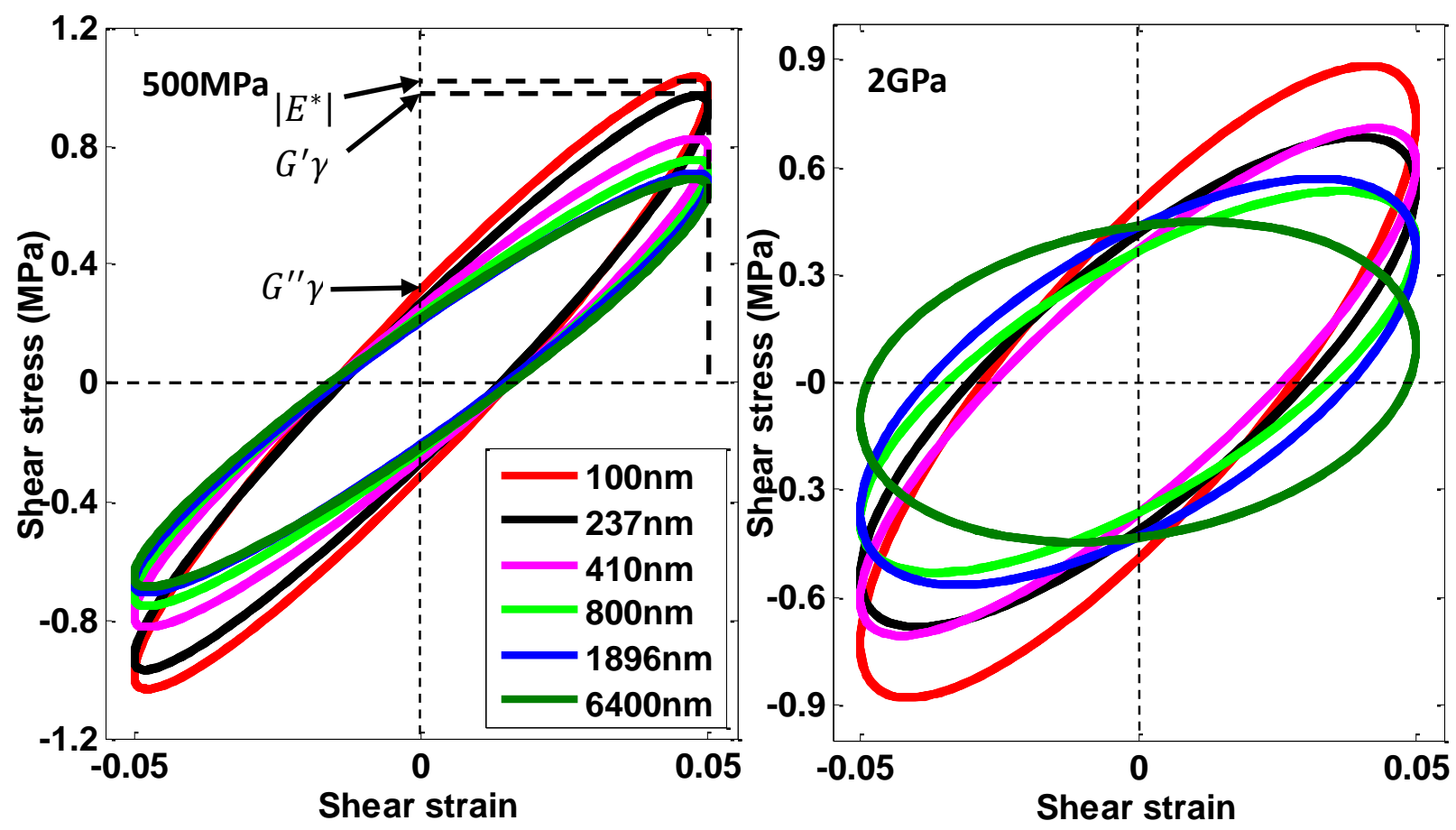

Figure 10: Stress versus strain of buckypapers made of different lengths of individual CNTs with shear frequencies $500 \mathrm{MPa}$ and $2 \mathbf{G H z}$ respectively. The notation in the left figure corresponds to the model with CNTs of length $100 \mathbf{~ n m}$. 


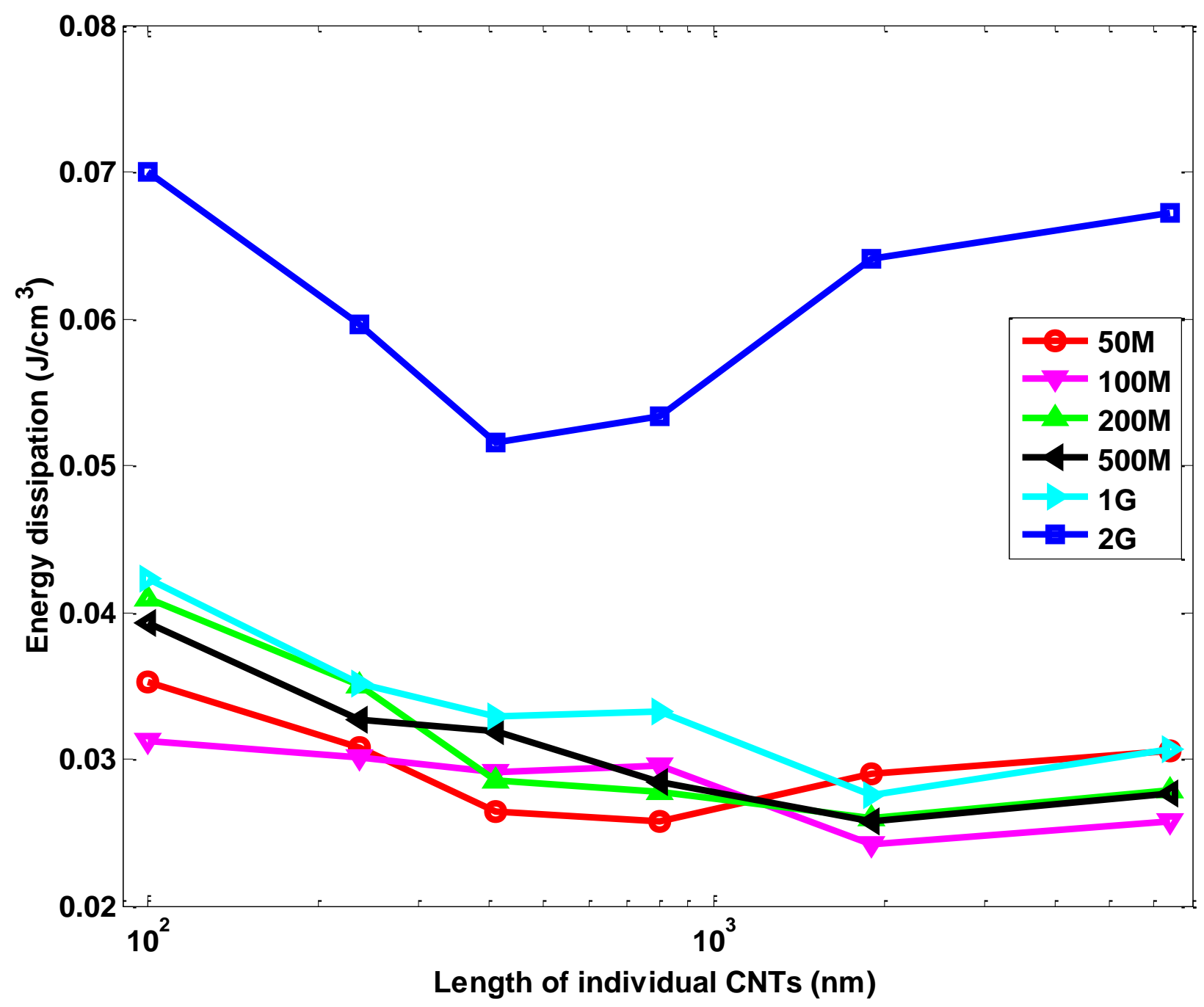

Figure 11: Energy dissipation density of as a function of the individual length of CNTs with shear frequencies ranging from $50 \mathrm{MHz}$ to $2 \mathrm{GHz}$. 